Supplementary Table 1. Differentially expressed genes and gene ontologies (GOs) from Figure 3.

\section{Upregulated genes}

\begin{tabular}{|c|}
\hline CEBPE \\
\hline TINAGL1 \\
\hline ANKRD22 \\
\hline GPR84 \\
\hline CHI3L1 \\
\hline CD177 \\
\hline SLCO4C1 \\
\hline OLFM4 \\
\hline C15orf48 \\
\hline LTF \\
\hline LCN2 \\
\hline PRTN3 \\
\hline ICA1 \\
\hline OLR1 \\
\hline MS4A3 \\
\hline MAPK13 \\
\hline S100A8 \\
\hline ANXA1 \\
\hline CTSG \\
\hline FPR2 \\
\hline PADI4 \\
\hline CDKN3 \\
\hline ELANE \\
\hline UPP1 \\
\hline ANXA3 \\
\hline CAMP \\
\hline ABCA13 \\
\hline RHOU \\
\hline ORM1 \\
\hline CHIT1 \\
\hline 5 -SeP \\
\hline S100A9 \\
\hline \\
\hline \\
\hline \\
\hline \\
\hline
\end{tabular}

\section{Downregulated Genes}

\begin{tabular}{|c|}
\hline LYPD6B \\
\hline ALS2CL \\
\hline TDRP \\
\hline TMIE \\
\hline ALPK1 \\
\hline RAMP3 \\
\hline VIPR1 \\
\hline LEF1 \\
\hline RYR3 \\
\hline CNGA1 \\
\hline ADH1C \\
\hline AMPD1 \\
\hline
\end{tabular}




\section{Upregulated GOs}

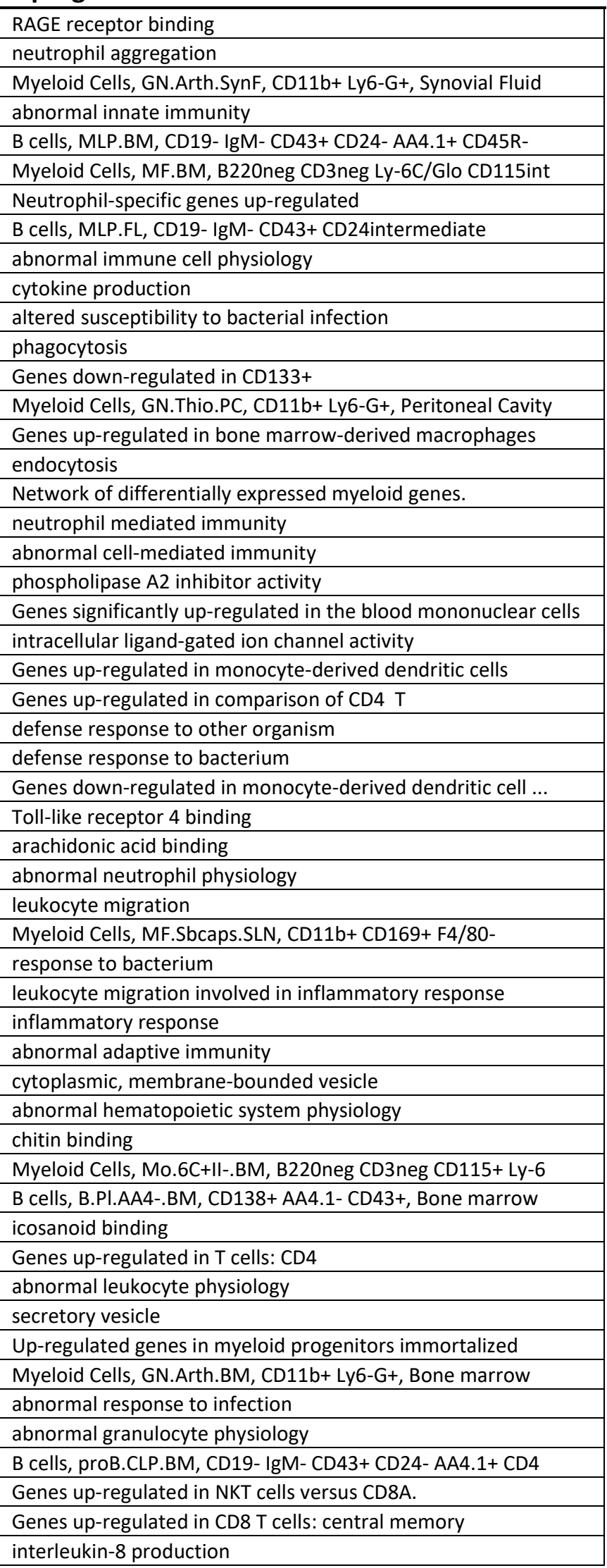

\section{Downregulated GOs}

Genes up-regulated in CD4 T conv over-expressing GATA1

alpha beta T cells, T.8Nve.Sp, 4- 8+ 25-62Lhi 44lo, Sp

Genes down-regulated in comparison of regulatory $T$ cell

Genes up-regulated in spleen B lymphocytes versus bone

Genes down-regulated in CD4 T conv over-expressing IKZF

alpha beta T cells, T.4.Pa.BDC, 4+ 8- BDC+, Pancreas, avg-2

Genes down-regulated in comparison of thymus regulatory

Genes down-regulated in comparison of TregLP versus Tconv

Genes down-regulated in comparison of lymph node

Genes down-regulated in CD8 T cells: naïve

Genes down-regulated in B lymphocytes: ZFX

Genes down-regulated in CD4 T conv over-expressing LEF1

Genes down-regulated in CD4 [GenelD=920] T cells

alpha beta T cells, T.4Nve.MLN, 4+ 8- 25-62Lhi 44lo,

CD positive, T.8Nve.Sp.OT1, CD8+ CD45.1+, Spleen,

Genes up-regulated in dendritic cells stimulated by LPS

Genes down-regulated in CXCR5+ BCL6+

Genes down-regulated in comparison of regulatory $T$ cell

alpha beta T cells, T.4Mem.LN, TCRb CD44high CD122lo CD

CD positive, CD4 Control, 4+8-B220-, Spleen,

Cluster P4 of genes with similar expression profiles

Genes down-regulated in comparison of TregCD103-KIrg1

Genes down-regulated in CD4 [GenelD=920] T conv

Genes up-regulated in CD4 [GeneID=920] versus granulocytes

Genes down-regulated in comparison of regulatory $T$ cell

Genes up-regulated in bone marrow-derived macrophages

Genes up-regulated in comparison of TconvLN versus Treg

alpha beta T cells, T.8Nve.MLN, 4- 8+ 25-62Lhi 44lo,

alpha beta T cells, T.4.PLN.BDC, 4+ 8- BDC+, Lymph Node 